\title{
The Application of VB GUI and Animation technology in the Automation Simulation Test
}

\author{
Ai-xin Yang ${ }^{1}$ \\ Xingtai University \\ Xingtai,Hebei,China \\ huozhiyi@sohu.com \\ Hong $\mathrm{Li}^{2}$ \\ Xingtai University \\ Xingtai,Hebei,China \\ xtwangqian01@163.com
}

\author{
Hong-yan $\mathrm{Liu}^{3}$ \\ Hengyang Finance Economics \\ and Industry Vocational College \\ Hengyang,Hunan,China \\ 3573198@qq.com
}

\author{
Qian Wang ${ }^{4}$ \\ Xingtai Polytechnic College \\ Xingtai,Hebei,China \\ 39563742@qq.com
}

\begin{abstract}
As the Internet is more and more widely used, the use of animation technology is increasingly popular. Microsoft GUI is an extension of the VB GUI, It provides a large number of writing classes and support codes and can deal with many standard Windows rogramming tasks,using VB GUI can simplify the programming and save a lot of coding work for evelopers.In the automation system development VB GUI and animation technology are used, and combining with the programming to realize, animation technology can meet requirements of the system function and greatly improve development efficiency and provide a new way to optimize designs for the automation system developers. In the article the author discusses how to combine VB technology with animation technology and do automation simulation and simulation tests, and expounds how to extract the simulation models.
\end{abstract}

Keywords: VB, animation technology, simulation, simulation test

\section{INTRODUCTION}

As the Internet is more and more widely used, the use of animation technology is increasingly popular. For example, the changes of the liquid level height and the liquid volume in the container or a fish swimming in the water are actually a kind of animation effects. When compiling configuration software, animation function is even more essential. The animation is realized by using humans' visual effects animation by alternately drawing a picture, then clearing the screen display part, and then drawing a second picture in the new position. VB works on the same principle, but VB does not require programmers to understand how pictures are reproduced and cleared, and all of the tedious work can be done by the special tools provided by VB. Therefore, VB can make both the method of making animation and its programming relatively simple ${ }^{[1]}$.

\section{INTRODUCTION TO VB TECHNOLOGY}

VB is a Microsoft application development tool offered by Microsoft Company and one of the most widely used programming languages. VB, with its graphical user interface development function and object-oriented programming method, belongs to the visual programming software package, and therefore can provide more development ideas for the developers. The simulation interface and the simulation and the test can be realized not only by using the graphical user interface GUI but also by combining GUI with VB object library.

Microsoft GUI is an extension of the VB GUI. It provides a large number of writing classes and support codes and can deal with many standard Windows programming tasks. Therefore, using VB GUI can simplify the programming and save a lot of coding work for developers. The animation technology is uniquely object oriented development environment with powerful functions, vivid animation and clear images. When applied in the automatic simulation system, it embodies many new features and can achieve functions which are difficult to realize in many of the original development environment, and therefore it can improve development efficiency. Animation technology represents the development direction of automatic simulation system and will definitely become the preferred technology among a new generation of application software ${ }^{[2]}$. Since Windows launched, the developers of the automation technology simulation system have been trying to find a new way to make its application programs both have the friendly interface of Windows and maintain their basic functions, but it is never satisfactory enough. The launch of VB GUI and the animation technology makes it possible. To explain the above-mentioned problems, the author, taking Visual Basic6.0 as an example, discusses in this article how to use animation techniques in VB GUI applications, and by combining VB GUI with the animation technology, develops perfect automation simulation application programs.

\section{The BASIC PRINCIPLES OF ANIMATION TECHNOLOGY}

In VB, animation making is very common. Animation technology consists of two parts: one is the object's movement relative to the screen, namely the screen level animation, and the other the object's internal movement, namely the relative symbols' animation. Animation is the sequential display of a series of images and graphics used to simulate movements, a set of related images displayed for a short interval of time, so the essence of animation are displayed images and rapid and clocked moving images. First draw a few pictures similar but having subtle changes, and then display in turn one of these pictures for a short interval of time. Because the time interval is very short, people can see continuous animation effects. In VB, image files of ico, bmp and 
wmf format can be put into memory by setting the picture attribute of the image or through the loadpicture function, the value returned by the function given to the picture attribute of the image object so as to be displayed in the image object. There are three ways to move or change images: images can be resized by modifying its wide and heigh attributes; images can be moved by using its move method, its left and top attributes properties indicating the current position of its left upper corner; load different images by using loadpicture function, and given to its picture attribute, and then different pictures will be displayed in the object, and changed images are realized this way. Timing control can be realized with the timer control and the time interval can be set by the interval attribute of the time object, that is, the time interval of the timer event process; the timer event is about what actions to do every interval set by the interval attribute. In the timer event process animation effects can be achieved by processing controlling the changes and movements of the image object. The interval value of the timer object decides the changing and moving speeds of the animation. The enable attribute of the timer object determines whether the timer event is effective: setting the enable attribute to true will start the timer events while setting to false will disable the timer event and the timer object is visible after running ${ }^{[3]}$.

\section{Common Problems When Animation TECHNOLOGY APPLIED IN VB GUI}

When applied VB GUI can be built not only with dynamic linear but also with dynamic curve, so, it can make full use of the advantages of animation technology. In its application, whether to choose the dynamic curve or the dynamic linear largely depends on the application programs' functions and the structure ratio of the different systems in the automation simulation development; the developer decides whether the superior performance brought about by dynamic linear is more important or the consistency of the automation simulation development system structure is more important. The VB GUI program with dynamic curve is more complicated, so, compared the program with dynamic linear, it has such disadvantages as low system efficiency and abnormal working and yet has the advantage of complete consistency between the simulated automation system and the real system ${ }^{[4]}$.

\section{ANIMATION TECHNOLOGY AND VB GUI APPLIED to AutOMATIC SimULATED SySTEM}

\section{A. System Initialization}

In VB GUI, to make an object move, you only need to stimulate its MOVE method and determine its movement direction and distance in that direction; this step is determined by the active source of correlation method and it is the power of the whole process, but it can only achieve one movement. In order to achieve continuous movements, a clock control will be needed. As is known to all, it is through a changed pulse that a clock control stimulates its movements and the pulse can be generated by the active source. Location changes of the active source can exactly meet the requirements for the clock control functions, so that you can realize continuous moving of the active source, and thus make the clock control work properly.

\section{B. Detemine the Algorithm}

Algorithm is mainly used to control the active source's movement scope, that is, to add constraints to the active source's movement, so that it can move only in a specified scope. Under specified conditions, its movement scope can approximate to our prescribed path through the approximation approach and the specific movement distances $\mathrm{C} 1$ and $\mathrm{C} 2$ are determined by the following algorithm:

$$
\begin{aligned}
& \mathrm{C} 1=(-1) \mathrm{X} 1(\mathrm{~B}[\mathrm{i}+1][0]-\mathrm{B}[\mathrm{i}][0]) /[\mathrm{M}] \\
& \mathrm{C} 2=(-1) \mathrm{X} 2(\mathrm{~B}[\mathrm{i}+1][0]-\mathrm{B}[\mathrm{i}][0]) /[\mathrm{M}]
\end{aligned}
$$

Array B [i] decides the target point and the path. Whether $\mathrm{X} 1$ and $\mathrm{X} 2$ are odd or even is related to the size of B [i] [0] and [i + 1] [0]] and B [i] [i] and B[i + 1] [i] and $\mathrm{M}$ is associated with the automatic system, so that it can make the active source move along a prescribed path $^{[5]}$.

\section{Process Discussion}

The passive source is controlled through the loop, functions and some curve equations. As the active source

keeps moving, its target point keeps changing. The changing target point is the new target point needed by

functions until the new target covers the path of a small number of values. As the target point constantly changes, another target point comes in succession after the previous target, and in this way the dynamic automatic simulation system is realized. So far, you can draw the flow chart of the whole automatic simulation system, as shown in figure 1 .

To distinguish the different structures, you can use different specified colors for the curve or the straight line. You can control the dynamic moving speeds of the active and passive sources by adjusting the clock pulse time interval ${ }^{[6]}$.

\section{Programs as examples}

Suppose you now need to draw a path after pressing the OK button of a window, you can determine the moving speed with the clock control in VB, then draw the flow chart and finally edit the code. The code is as follows:

Private Sub Timerl_Timer ()

Dim A (0 to $n)$ as integer

Dim B (0 to n) (0 to n) as integer 
For $\mathrm{i}=0$ to $\mathrm{n}$

Sub1

$\mathrm{i}=\mathrm{i}+1$

Next

End Sub

Private Sub1 ()

if $\mathrm{A}[\mathrm{i}]=\mathrm{Line} 1$ then

Sub2

Else if $\mathrm{A}[\mathrm{i}]=\sin \mathrm{x}$ then

Sub3

End Sub

Private Sub2 ()

img.Left $=B[i][0]$

img.Top= B[i][1]

img.move img.Left+C1, img.Top+C2

End if

End Sub

Private Sub3 ()

img.Left $=B[i][0]$

img.Top $=\mathrm{B}[\mathrm{i}][1]$

$Y \min =1 \mathrm{E}+101$

$\mathrm{Ymax}=-1 \mathrm{E}+101$

$\mathrm{Xmin}=2$

$\mathrm{X} \max =10$

Form 1.Cls

Form l. Scale Mode= -3

Xpixels $=$ Form l. Scale Width -1

For i= 1 To Xpixels
$\mathrm{t}=\mathrm{Xmin}+(\mathrm{Xmax}-\mathrm{Xmin}) * \mathrm{i} / \mathrm{XpiXels}$

Functional $=$ functional $(\mathrm{t})$

if functional>Ymax then

Ymax $=$ functional

if functional $<$ Ymin then

Ymin= functional

Next

Form1.Scale (Xmin,Ymin)-(Xmax,Ymax)

Form1.ForeC olor $=$ RGB $(0,0,225)$

Form1.PSet (Xmin, functional (Xmin))

For $\mathrm{i}=0$ to Xpixels

$\mathrm{t}=\mathrm{Xmin}+(\mathrm{Xmax}-\mathrm{Xmin}) * \mathrm{i} / \mathrm{Xpixels}$

Form1.Line $=(\mathrm{t}$, functional $(\mathrm{t}))$

Next

End Sub

Functional (ByVal X as Double) as Double

Functional $=\operatorname{Exp}(2 / \mathrm{X}) * \cos (2 * \mathrm{X})$

End function

\section{CONCLUSIONS}

In the automation system development VB GUI and animation technology are used, and combining with the programming to realize. By adopting object oriented and event-driven programming principle and making use of humans' visional persistence, making a series of images displayed continuously can produce animation effects in developing application programs. Practice has proved that programming of Windows style can be realized by $\mathrm{VB}$, and that the internal system can be reached by using key frames in the animation technology ${ }^{[7]}$. It can meet requirements of the system function and greatly improve development efficiency and provide a new way to optimize designs for the automation system developers.

\section{REFERENCES}

- [1] Zhang Yue, "Development and outlook of virtual reality technology,”J.The computer world,5(2011),pp.37-38. 
- [2] Zhang Conglin, “Computer aided design,”J.The computer world,6(2010),pp.72-74.

- [3] Huang Keli, “System simulation technology,”M.National university of defense technology press, (2011),pp.105-107.

- [4] Hu Feng,Sun Guoji, “Dynamic system computer simulation technology were reviewed," The computer simulation,J.(3)2010,pp.21-23.

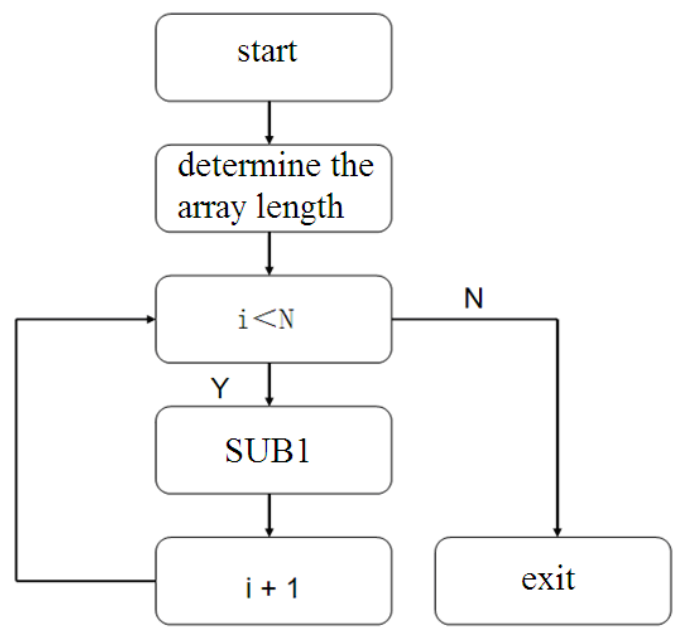

- [5] Wang Chengwen, "The implementation and application of virtual reality technology,” J. Modern military ,5(2011),pp.87-90.

- [6] Jiang Qinquan, "VR technology development, ”Modern defense technology, J.(1)2010,pp.46-48.

- [7] Zhao Li, "Virtual reality technology is a key technology of the 21st century", Command and control system of the ship, J.(4)2011,pp.98-99.

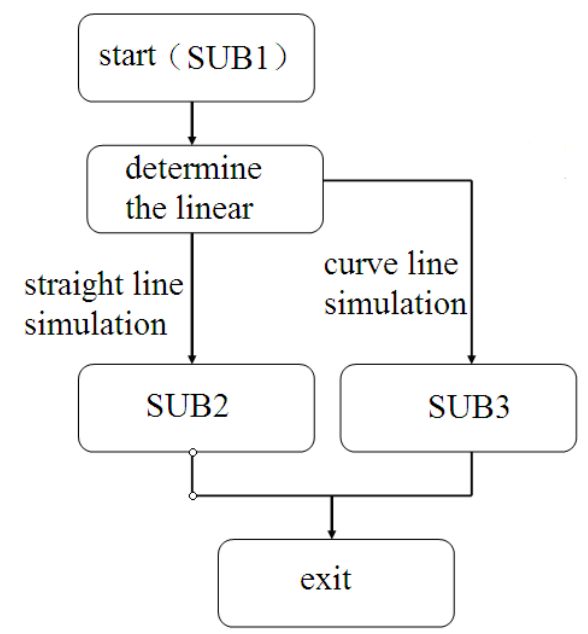

Figure 1 Flow chart of the automatic simulation system 